\title{
FLORENT BERTHELIN
}

\section{FRANÇOIS BOUCHUT}

\section{Solution with finite energy to a BGK system relaxing to isentropic gas dynamics}

Annales de la faculté des sciences de Toulouse $6^{e}$ série, tome $9, \mathrm{n}^{\circ} 4$ (2000), p. 605-630

<http://www.numdam.org/item?id=AFST_2000_6_9_4_605_0>

(C) Université Paul Sabatier, 2000, tous droits réservés.

L'accès aux archives de la revue «Annales de la faculté des sciences de Toulouse » (http://picard.ups-tlse.fr/ annales/) implique l'accord avec les conditions générales d'utilisation (http://www.numdam.org/conditions). Toute utilisation commerciale ou impression systématique est constitutive d'une infraction pénale. Toute copie ou impression de ce fichier doit contenir la présente mention de copyright.

\section{NumDam}

Article numérisé dans le cadre du programme Numérisation de documents anciens mathématiques http://www.numdam.org/ 


\title{
Solution with finite energy to a BGK system relaxing to isentropic gas dynamics ${ }^{(*)}$
}

\author{
Florent Berthelin ${ }^{(1)}$ and François Bouchut ${ }^{(2)}$
}

RÉSUMÉ. - On considère une équation BGK cinétique vectorielle donnant la dynamique des gaz isentropique dans la limite de relaxation. Nous montrons l'existence d'une solution faible satisfaisant une inégalité d'entropie cinétique, pour toute donnée initiale d'énergie finie.

Abstract. - We consider a vector kinetic BGK equation leading to isentropic gas dynamics in the relaxation limit. We prove the existence of a weak solution satisfying a kinetic entropy inequality for any initial data with finite energy.

\section{TABLE OF CONTENTS}

1. Introduction and main results

2. Properties of the kinetic entropy

3. Stability

4. Existence of a solution

5. Entropy equation

(*) Reçu le 4 juillet 2000, accepté le 4 décembre 2000

(1) Université d'Orléans et CNRS, UMR 6628, Département de Mathématiques, B.P. 6759,45067 Orléans cedex 2, France. e-mail: florent.berthelin@labomath.univ-orleans.fr (2) Département de Mathématiques et Applications, École Normale Supérieure et CNRS, UMR 8553, 45, rue d'Ulm, 75230 Paris cedex 05, France.

e-mail:Francois.Bouchut@ens.fr 


\section{Introduction and main results}

Relaxation models such as proposed in [6] have proved their efficiency in building numerical methods for conservation laws that are very easy to code and have nice properties. General strategies have been proposed in [9], [13], [1], [3] to build BGK approaches for systems of conservation laws. However, analysis of such models has mainly be achieved until now only when the relaxed equation is scalar (see [14]). Here we consider a BGK model for the one-dimensional system of isentropic gas dynamics

$$
\left\{\begin{array}{c}
\partial_{t} \rho+\partial_{x}(\rho u)=0, \\
\partial_{t}(\rho u)+\partial_{x}\left(\rho u^{2}+\kappa \rho^{\gamma}\right)=0,
\end{array}\right.
$$

with $\rho(t, x) \geqslant 0, u(t, x) \in \mathbb{R}$ and $\kappa>0,1<\gamma<3$. The kinetic model has been introduced in [3] and can be written

$$
\left.\partial_{t} f+\xi \partial_{x} f=\frac{M[f]-f}{\varepsilon} \quad \text { in }\right] 0, \infty[\times \mathbb{R} \times \mathbb{R},
$$

where $f=f(t, x, \xi) \in \mathbb{R}^{2}, t \geqslant 0, x \in \mathbb{R}, \xi \in \mathbb{R}$,

$$
\begin{gathered}
f(t, x, \xi) \in D, \\
D=\left\{\left(f_{0}, f_{1}\right) \in \mathbb{R}^{2}, f_{0}>0 \text { or } f_{1}=f_{0}=0\right\}, \\
M[f](t, x, \xi)=M(\rho(t, x), u(t, x), \xi), \\
\rho(t, x)=\int_{\mathbb{R}} f_{0}(t, x, \xi) d \xi, \rho(t, x) u(t, x)=\int_{\mathbb{R}} f_{1}(t, x, \xi) d \xi,
\end{gathered}
$$

and

$$
\begin{gathered}
M(\rho, u, \xi)=(\chi(\rho, \xi-u),((1-\theta) u+\theta \xi) \chi(\rho, \xi-u)) \\
\chi(\rho, \xi)=c_{\gamma, \kappa}\left(\frac{4 \gamma \kappa}{(\gamma-1)^{2}} \rho^{\gamma-1}-\xi^{2}\right)_{+}^{\lambda}, \\
\theta=\frac{\gamma-1}{2}, \lambda=\frac{1}{\gamma-1}-\frac{1}{2}, c_{\gamma, \kappa}=\frac{1}{J_{\lambda}}\left(\frac{4 \gamma \kappa}{(\gamma-1)^{2}}\right)^{-1 /(\gamma-1)}, \\
J_{\lambda}=\int_{-1}^{1}\left(1-z^{2}\right)^{\lambda} d z=\sqrt{\pi} \Gamma(\lambda+1) / \Gamma(\lambda+3 / 2) .
\end{gathered}
$$

The kinetic equilibrium $\chi$ in (1.8) has been introduced in [7] as a generating function for entropies, and has also been used in the stability analysis of [10]. This function is also involved in [12] for the so called kinetic formulation, which is different from the BGK equation considered here. Previous results 
for scalar equations can be found in [4], [11], [17] (see also [5]). The first existence result for a BGK equation was given in [15] (see also [16]).

The main result of this paper is the existence of a global solution to the system (1.2) with initial data

$$
f(0, x, \xi)=f^{0}(x, \xi)
$$

satisfying energy bounds. We recall that the energy for (1.1) is given by

$$
\eta(\rho, u)=\rho u^{2} / 2+\frac{\kappa}{\gamma-1} \rho^{\gamma},
$$

and is a mathematical entropy for (1.1). The corresponding kinetic entropy for (1.2) is given, for $f=\left(f_{0}, f_{1}\right) \in D$, by

$$
\begin{gathered}
H(f, \xi)=\frac{\theta}{1-\theta} \frac{\xi^{2}}{2} f_{0}+\frac{\theta}{2 c_{\gamma, \kappa}^{1 / \lambda}} \frac{f_{0}^{1+1 / \lambda}}{1+1 / \lambda}+\frac{1}{1-\theta} \frac{1}{2} \frac{f_{1}^{2}}{f_{0}}-\frac{\theta}{1-\theta} \xi f_{1} \\
=\frac{\theta}{1-\theta} \frac{1}{2}\left(\frac{f_{1}}{\sqrt{f_{0}}}-\xi \sqrt{f_{0}}\right)^{2}+\frac{\theta}{2 c_{\gamma, \kappa}^{1 / \lambda}} \frac{f_{0}^{1+1 / \lambda}}{1+1 / \lambda}+\frac{1}{2} \frac{f_{1}^{2}}{f_{0}} \geqslant 0, \\
H(0, \xi)=0 .
\end{gathered}
$$

We have the following theorems.

Theorem 1.1. - Assume that $f^{0} \in L^{1}\left(\mathbb{R}_{x} \times \mathbb{R}_{\xi}\right)$ satisfies

$$
\begin{gathered}
f^{0}(x, \xi) \in D \text { a.e. in } \mathbb{R} \times \mathbb{R}, \\
\iint_{\mathbb{R} \times \mathbb{R}} H\left(f^{0}(x, \xi), \xi\right) d x d \xi=C_{H}^{0}<\infty,
\end{gathered}
$$

and

$$
\iint_{\mathbb{R} \times \mathbb{R}} x^{2}\left(f^{0}\right)_{0}(x, \xi) d x d \xi=C_{\Delta}^{0}<\infty .
$$

Then there exists a solution $f$ to (1.2)-(1.11) satisfying

$$
\begin{gathered}
f \in C\left(\left[0, \infty\left[, L^{1}(\mathbb{R} \times \mathbb{R})\right),\right.\right. \\
f \in L^{\infty}\left(\left[0, \infty\left[, L^{1}(\mathbb{R} \times \mathbb{R})\right),\right.\right. \\
\forall t \geqslant 0, f(t, x, \xi) \in D \text { a.e. in } \mathbb{R} \times \mathbb{R}, \\
\partial_{t}\left(\int_{\mathbb{R}} f d \xi\right)+\partial_{x}\left(\int_{\mathbb{R}} \xi f d \xi\right)=0, \\
-607-
\end{gathered}
$$


Florent Berthelin, François Bouchut

$$
\begin{gathered}
\forall t \geqslant 0, \iint_{\mathbb{R} \times \mathbb{R}} f(t, x, \xi) d x d \xi=\iint_{\mathbb{R} \times \mathbb{R}} f^{0}(x, \xi) d x d \xi, \\
\forall t \geqslant 0, \iint_{\mathbb{R} \times \mathbb{R}} H(f(t, x, \xi), \xi) d x d \xi \leqslant C_{H}^{0}, \\
\forall t \geqslant 0, \iint_{\mathbb{R} \times \mathbb{R}} x^{2} f_{0}(t, x, \xi) d x d \xi \leqslant \max \left(C_{\Delta}^{0}, \frac{4 \varepsilon^{2}}{\theta} C_{H}^{0}\right)(1+t / \varepsilon)^{2} .
\end{gathered}
$$

THEOREM 1.2. - If (1.17) is not satisfied, we have the same result except (1.24).

To obtain this result, we have to work with the Tychonoff-Schauder theorem instead of the usual Schauder theorem. The only significant change is for compactness and will be explain in the corresponding section.

THEOREM 1.3. - The solution $f$ obtained in Theorems 1.1 or 1.2 satisfies

$$
\begin{gathered}
H(f, \xi) \in C\left(\left[0, \infty\left[, L^{1}(\mathbb{R} \times \mathbb{R})\right),\right.\right. \\
\partial_{t}(H(f, \xi))+\xi \partial_{x}(H(f, \xi))=\frac{1}{\varepsilon} H^{\prime}(f, \xi) \cdot(M[f]-f),
\end{gathered}
$$

with

$$
H^{\prime}(f, \xi)=\left(\frac{\theta}{1-\theta} \frac{\xi^{2}}{2}+\frac{\theta}{2 c_{\gamma, \kappa}^{1 / \lambda}} f_{0}^{1 / \lambda}-\frac{1}{1-\theta} \frac{1}{2} \frac{f_{1}^{2}}{f_{0}^{2}},-\frac{\theta}{1-\theta} \xi+\frac{1}{1-\theta} \frac{f_{1}}{f_{0}}\right)
$$

for $f \neq 0$, and $H^{\prime}(0, \xi)=\left(\frac{\theta}{1-\theta} \frac{\xi^{2}}{2},-\frac{\theta}{1-\theta} \xi\right)$ by convention. Moreover,

$$
H^{\prime}(f, \xi) \cdot(M[f]-f) \in L^{1}(] 0, T[\times \mathbb{R} \times \mathbb{R}) \quad \text { for any } T>0
$$

as well as each term from the decomposition (1.27). Besides, $\xi H(f(t, x, \xi), \xi) \in$ $L^{1}(] 0, T\left[\times B_{R} \times \mathbb{R}\right)$ for any $T>0, R>0$,

$$
\begin{aligned}
& \frac{\partial}{\partial t} \int_{\mathbb{R}} H(f(t, x, \xi), \xi) d \xi+\frac{\partial}{\partial x} \int_{\mathbb{R}} \xi H(f(t, x, \xi), \xi) d \xi \\
&= \frac{1}{\varepsilon} \int_{\mathbb{R}}\left(H^{\prime}(f, \xi)-\left(\frac{\gamma \kappa}{\gamma-1} \rho^{\gamma-1}-\frac{u^{2}}{2}, u\right)\right) \cdot(M[f]-f) d \xi \leqslant 0, \\
&-608-
\end{aligned}
$$




$$
\begin{aligned}
& \frac{d}{d t} \iint_{\mathbb{R} \times \mathbb{R}} H(f(t, x, \xi), \xi) d x d \xi \\
= & \frac{1}{\varepsilon} \iint_{\mathbb{R} \times \mathbb{R}}\left(H^{\prime}(f, \xi)-\left(\frac{\gamma \kappa}{\gamma-1} \rho^{\gamma-1}-\frac{u^{2}}{2}, u\right)\right) \cdot(M[f]-f) d x d \xi \leqslant 0,
\end{aligned}
$$

and the integrand in the right-hand sides of (1.29) and (1.30) is nonpositive.

Let us finally mention that we can study kinetic invariant domains and establish the relaxation limit to (1.1), this is done in [2].

\section{Properties of the kinetic entropy}

We recall the value of the moments of $M$,

$$
\begin{gathered}
\int_{\mathbb{R}} M(\rho, u, \xi) d \xi=(\rho, \rho u), \\
\int_{\mathbb{R}} \xi M(\rho, u, \xi) d \xi=\left(\rho u, \rho u^{2}+\kappa \rho^{\gamma}\right),
\end{gathered}
$$

and

$$
\int_{\mathbb{R}} \frac{1}{2} \xi^{2} M_{0}(\rho, u, \xi) d \xi=\frac{1}{2} \rho u^{2}+\frac{\kappa}{\gamma-1} \rho^{\gamma}=\eta(\rho, u)
$$

for every $\rho \geqslant 0$ and $u \in \mathbb{R}$. We have the following identities which link the energy and the kinetic entropy. They can be obtained easily.

Proposition 2.1. - The energy $\eta$ of (1.12) and the kinetic entropy $H$ of (1.13) satisfy

$$
\int_{\mathbb{R}} H(M(\rho, u, \xi), \xi) d \xi=\eta(\rho, u) \quad \text { for every } \rho \geqslant 0, u \in \mathbb{R},
$$

and

$$
\left\{\begin{array}{l}
\frac{\partial H}{\partial f_{0}}(M(\rho, u, \xi), \xi)=\frac{\partial \eta}{\partial \rho}(\rho, u)=\frac{\gamma \kappa}{\gamma-1} \rho^{\gamma-1}-u^{2} / 2, \\
\frac{\partial H}{\partial f_{1}}(M(\rho, u, \xi), \xi)=\frac{\partial \eta}{\partial(\rho u)}(\rho, u)=u,
\end{array}\right.
$$

for every $\rho>0, u \in \mathbb{R}$ and $\xi \in \mathbb{R}$ such that $M_{0}(\rho, u, \xi)>0$.

The function $H$ has the following properties.

Proposition 2.2. - i) $H$ is convex with respect to $f$ in $D$,

ii) $H$ is continuous with respect to $f$ in $\Gamma_{A}=\left\{\left(f_{0}, f_{1}\right) \in D ;\left|f_{1}\right| \leqslant A f_{0}\right\}$ for any $A>0$. 
Proof. $-i$ ) In $] 0, \infty[\times \mathbb{R}$, it is easy to compute the hessian and to check its nonnegativity. Then, on a straight line from the origin, $f_{1}^{2} / f_{0}$ is linear, thus $H$ is convex in $D$.

ii) The only difficulty is at 0 . Let $\left(f^{n}\right)_{n \geqslant 0}$ be a sequence in $\Gamma_{A} \backslash\{0\}$ which converges to 0 . Then

$$
\left|\frac{\left(f^{n}\right)_{1}^{2}}{\left(f^{n}\right)_{0}}\right| \leqslant A^{2}\left(f^{n}\right)_{0} \underset{n \rightarrow \infty}{\longrightarrow} 0,
$$

thus $\mathrm{H}$ is continuous at 0 in $\Gamma_{A}$.

Lemma 2.3 (Coerciveness). - There exist $\varepsilon_{0}, \varepsilon_{1}>0$ such that for any $f \in D, \xi \in \mathbb{R}$, we have

$$
H(f, \xi) \geqslant \varepsilon_{0} f_{0}^{p_{0}}+\varepsilon_{1}\left|f_{1}\right|^{p_{1}},
$$

with

$$
p_{0}=1+1 / \lambda>1, \quad p_{1}=2(1+\lambda) /(1+2 \lambda)>1 .
$$

Proof. - Let $f \in D, f \neq 0$. From identity (1.13), we deduce that

$$
H(f, \xi) \geqslant \frac{\theta}{2 c_{\gamma, \kappa}^{1 / \lambda}} \frac{f_{0}^{1+1 / \lambda}}{1+1 / \lambda}+\frac{1}{2} \frac{f_{1}^{2}}{f_{0}} .
$$

We have $\left|f_{1}\right|^{p_{1}}=\left(\frac{\left|f_{1}\right|}{\sqrt{f_{0}}}\right)^{p_{1}}\left(\sqrt{f_{0}}\right)^{p_{1}}$, and using Young's inequality,

$$
\left|f_{1}\right|^{p_{1}} \leqslant \frac{p_{1}}{2}\left(\frac{\left|f_{1}\right|}{\sqrt{f_{0}}}\right)^{2}+\frac{p_{1}}{2+2 / \lambda} f_{0}^{1+1 / \lambda} .
$$

Taking $0<\varepsilon<1$ such that $\varepsilon<\theta / c_{\gamma, \kappa}^{1 / \lambda}$, and setting $\varepsilon_{0}=\left(\theta / c_{\gamma, \kappa}^{1 / \lambda}-\varepsilon\right) /(2+$ $2 / \lambda)$ and $\varepsilon_{1}=\varepsilon / p_{1}$, we get

$$
\begin{aligned}
H(f, \xi) & \geqslant \frac{\theta}{2 c_{\gamma, \kappa}^{1 / \lambda}} \frac{f_{0}^{1+1 / \lambda}}{1+1 / \lambda}+\varepsilon \frac{1}{2} \frac{f_{1}^{2}}{f_{0}} \\
& \geqslant \frac{\theta}{2 c_{\gamma, \kappa}^{1 / \lambda}} \frac{f_{0}^{1+1 / \lambda}}{1+1 / \lambda}-\frac{\varepsilon}{2+2 / \lambda} f_{0}^{1+1 / \lambda}+\frac{\varepsilon}{p_{1}}\left|f_{1}\right|^{p_{1}} \\
& =\varepsilon_{0}{f_{0}}^{p_{0}}+\varepsilon_{1}\left|f_{1}\right|^{p_{1}} .
\end{aligned}
$$

The key result of this section is the following. 
Proposition 2.4 (Key Convexity inequality). - For any $f \in D$, $\rho \geqslant 0$ and $u, \xi \in \mathbb{R}$, we have

$$
H(f, \xi) \geqslant H(M(\rho, u, \xi), \xi)+\left(\frac{\gamma \kappa}{\gamma-1} \rho^{\gamma-1}-\frac{u^{2}}{2}, u\right) \cdot(f-M(\rho, u, \xi)) .
$$

Proof. - Let us consider $\psi: D \rightarrow \mathbb{R}$ defined by $\psi(f)=H(f, \xi)-H(M(\rho, u, \xi), \xi)-\left(\frac{\gamma \kappa}{\gamma-1} \rho^{\gamma-1}-\frac{u^{2}}{2}, u\right) \cdot(f-M(\rho, u, \xi))$, which is possible because $M(\rho, u, \xi) \in D$. We have to prove that $\psi \geqslant 0$.

- At infinity, by Lemma $2.3, \psi(f) \geqslant \varepsilon_{0} f_{0}{ }^{p_{0}}+\varepsilon_{1}\left|f_{1}\right|^{p_{1}}+a f_{0}+b f_{1}+e$ with $a, b, e \in \mathbb{R}$, and since $p_{0}, p_{1}>1, \psi(f) \rightarrow \infty$ when $f_{0}+\left|f_{1}\right|$ tends to infinity. Moreover, $\psi(f) \geqslant a f_{0}+b f_{1}+e$, and we deduce that $\psi$ is lower bounded in $D$.

- At the frontier $\left\{f_{0}=0, f_{1} \neq 0\right\}$, $f_{1}^{2} / f_{0} \rightarrow \infty$ and $\psi(f)$ also.

- At 0 , If $M_{0}(\rho, u, \xi)>0$, then by Proposition 2.2(i) and (2.5), (2.8) holds true for any $f \in D$, thus $\varliminf_{f \rightarrow 0} \psi(f) \geqslant 0$. On the contrary, if $M_{0}(\rho, u, \xi)=0$, then

$$
\psi(f)=H(f, \xi)-\left(\frac{\gamma \kappa}{\gamma-1} \rho^{\gamma-1}-u^{2} / 2, u\right) \cdot f .
$$

Since $H(f, \xi) \geqslant 0$, this yields $\varliminf_{f \rightarrow 0} \psi(f) \geqslant 0$, in any case.

- Inside $D$

It only remains to study possible minima of $\psi$ in $\operatorname{int}(D)$. But $\psi$ is smooth there, and if $f_{0}>0$,

$$
\frac{\partial \psi}{\partial f_{0}}(f, \xi)=\frac{\theta}{1-\theta} \frac{\xi^{2}}{2}+\frac{\theta}{2 c_{\gamma, \kappa}^{1 / \lambda}} f_{0}^{1 / \lambda}-\frac{1}{1-\theta} \frac{1}{2}\left(\frac{f_{1}}{f_{0}}\right)^{2}-\frac{\gamma \kappa}{\gamma-1} \rho^{\gamma-1}+\frac{u^{2}}{2},
$$

and

$$
\frac{\partial \psi}{\partial f_{1}}(f, \xi)=\frac{1}{1-\theta} \frac{f_{1}}{f_{0}}-\frac{\theta}{1-\theta} \xi-u .
$$

If there is a minimum of $\psi$ in $\operatorname{int}(D)$, at this point, its partial derivatives must vanish, thus

$$
\frac{f_{1}}{f_{0}}=(1-\theta) u+\theta \xi
$$


and

$$
\begin{aligned}
\frac{\theta}{2 c_{\gamma, \kappa}^{1 / \lambda}} f_{0}^{1 / \lambda} & =\frac{1}{1-\theta} \frac{1}{2}\left(\frac{f_{1}}{f_{0}}\right)^{2}+\frac{\gamma \kappa}{\gamma-1} \rho^{\gamma-1}-\frac{u^{2}}{2}-\frac{\theta}{1-\theta} \frac{\xi^{2}}{2} \\
& =\frac{\theta}{2}\left(\frac{4 \gamma \kappa}{(\gamma-1)^{2}} \rho^{\gamma-1}-(\xi-u)^{2}\right) .
\end{aligned}
$$

We deduce that $M_{0}(\rho, u, \xi)>0$ and $f=M(\rho, u, \xi)$. The value of $\psi$ at this point is 0 .

Putting all the steps together, we conclude that $\psi \geqslant 0$ in $D$.

An important consequence of this result is the entropy minimization principle.

Proposition 2.5. - Consider $f \in L^{1}\left(\mathbb{R}_{\xi}\right)$ such that $f \in D$ a.e. and

$$
\int_{\mathbb{R}} H(f(\xi), \xi) d \xi<\infty .
$$

Then

$$
\int_{\mathbb{R}} H(M[f](\xi), \xi) d \xi \leqslant \int_{\mathbb{R}} H(f(\xi), \xi) d \xi
$$

Proof. - Take in (2.8)

$$
\rho=\int_{\mathbb{R}} f_{0}(\xi) d \xi, \quad u=\frac{\int_{\mathbb{R}} f_{1}(\xi) d \xi}{\int_{\mathbb{R}} f_{0}(\xi) d \xi}, \quad \text { or } u=0 \text { if } \rho=0 .
$$

Then $M[f](\xi)=M(\rho, u, \xi)$, the terms of the right-hand side are integrable with respect to $\xi$, and $\int_{\mathbb{R}} M[f](\xi) d \xi=\int_{\mathbb{R}} f(\xi) d \xi$. Thus the result is obtained by integration in $\xi$.

We can also deduce that the entropy dissipation in (1.29) has a sign.

Proposition 2.6. - For any $f \in D, \rho \geqslant 0, u, \xi \in \mathbb{R}$,

$$
\left(H^{\prime}(f, \xi)-\left(\frac{\gamma \kappa}{\gamma-1} \rho^{\gamma-1}-\frac{u^{2}}{2}, u\right)\right) \cdot(f-M(\rho, u, \xi)) \geqslant 0 .
$$

Proof. - Since $H$ is convex in $D$, continuous in the cones of Proposition 2.2 and smooth in $\operatorname{int}(D)$, we have if $f \neq 0$

$$
H(M(\rho, u, \xi), \xi) \geqslant H(f, \xi)+H^{\prime}(f, \xi)(M(\rho, u, \xi)-f) .
$$


Letting $f \rightarrow 0$ on the axis $f_{1}=0$, we obtain using (1.27) that (2.10) is indeed true for any $f \in D$ with the convention

$$
H^{\prime}(0, \xi)=\left(\frac{\theta}{1-\theta} \frac{\xi^{2}}{2},-\frac{\theta}{1-\theta} \xi\right) .
$$

The case $f=0$ can also be seen directly by the definition of $H$. Finally, by adding (2.10) to (2.8), we get (2.9).

Let us end this part by estimates deduced from the boundedness of the kinetic entropy.

Proposition 2.7. - Consider $f \in L^{1}\left(\mathbb{R}_{x} \times \mathbb{R}_{\xi}\right)$ such that $f \in D$ a.e., and

$$
\begin{gathered}
\iint_{\mathbb{R} \times \mathbb{R}} H(f(x, \xi), \xi) d x d \xi \leqslant C_{H}, \\
\iint_{\mathbb{R} \times \mathbb{R}} f_{0}(x, \xi) d x d \xi \leqslant C_{0} .
\end{gathered}
$$

Then

$$
\begin{gathered}
\iint_{\mathbb{R} \times \mathbb{R}}\left|f_{1}(x, \xi)\right| d x d \xi \leqslant \sqrt{2 C_{0} C_{H}}, \\
\iint_{\mathbb{R} \times \mathbb{R}} \xi^{2} f_{0}(x, \xi) d x d \xi \leqslant \frac{4}{\theta} C_{H}, \\
\iint_{\mathbb{R} \times \mathbb{R}}|\xi|\left|f_{1}(x, \xi)\right| d x d \xi \leqslant \sqrt{\frac{8}{\theta}} C_{H}, \\
f_{i} \text { is bounded in } L^{p_{i}}(\mathbb{R} \times \mathbb{R}) \text { for } i=0,1 .
\end{gathered}
$$

Proof. - We recall that $p_{0}, p_{1}$ are defined by (2.7). First, using (1.13), we have

$$
\mathbf{I}_{f_{0}>0} \frac{f_{1}^{2}}{f_{0}} \leqslant 2 H(f, \xi) .
$$

Then, since $f_{1}$ vanishes where $f_{0}=0$,

$$
\begin{gathered}
\left|f_{1}\right|=\left|\mathbf{I}_{f_{0}>0} f_{1}\right|=\left|\mathbf{I}_{f_{0}>0} \frac{f_{1}}{\sqrt{f_{0}}} \times \sqrt{f_{0}}\right| \leqslant \sqrt{2 H(f, \xi) \times f_{0}} . \\
-613-
\end{gathered}
$$


Thus, from the Cauchy-Schwarz inequality we obtain (2.12). Next, with (1.13) again,

$$
\begin{aligned}
\xi^{2} f_{0} & =\left|\mathbf{I}_{f_{0}>0} \frac{f_{1}}{\sqrt{f_{0}}}-\xi \sqrt{f_{0}}-\mathbf{I}_{f_{0}>0} \frac{f_{1}}{\sqrt{f_{0}}}\right|^{2} \\
& \leqslant\left(\left|\mathbf{I}_{f_{0}>0} \frac{f_{1}}{\sqrt{f_{0}}}-\xi \sqrt{f_{0}}\right|+\left|\mathbf{I}_{f_{0}>0} \frac{f_{1}}{\sqrt{f_{0}}}\right|\right)^{2} \\
& \leqslant 2\left[\left(\mathbf{I}_{f_{0}>0} \frac{f_{1}}{\sqrt{f_{0}}}-\xi \sqrt{f_{0}}\right)^{2}+\mathbf{I}_{f_{0}>0} \frac{f_{1}^{2}}{f_{0}}\right] \\
& \leqslant 2\left[\frac{2(1-\theta)}{\theta} H(f, \xi)+2 H(f, \xi)\right] \\
& =\frac{4}{\theta} H(f, \xi),
\end{aligned}
$$

and (2.13) follows. Then, we write

$$
\left|\mathbf{I}_{f_{0}>0} \xi f_{1}\right|=\left|\left(\mathbf{I}_{f_{0}>0} f_{1} / \sqrt{f_{0}}\right) \times\left(\xi \sqrt{f_{0}}\right)\right| \leqslant \sqrt{2 H(f, \xi)} \times \sqrt{\frac{4}{\theta} H(f, \xi)},
$$

and obtain (2.14). Finally, (2.15) follows obviously from Lemma 2.3.

\section{Stability}

In this section, we extend the analysis of [15] and prove the stability of an approximate solution. We shall denote by $\varphi_{n}(x, \xi) \rightarrow \varphi(x, \xi)$ in $L^{1}\left(\mathbb{R}_{l o c} \times \mathbb{R}\right)$ the convergence in $L^{1}(\omega \times \mathbb{R})$ for any $\omega \subset \subset \mathbb{R}$, and we shall use several similar conventions.

Let us first recall the classical characteristics formula for (1.2).

Lemma 3.1. - Let $h \in L^{1}(] 0, T\left[, L^{1}(\mathbb{R} \times \mathbb{R})\right)$ and $f^{0} \in L^{1}(\mathbb{R} \times \mathbb{R})$. Then there exists a unique solution

$$
f \in C\left([0, T], L^{1}(\mathbb{R} \times \mathbb{R})\right)
$$

to the problem

$$
\left\{\begin{array}{c}
\partial_{t} f+\xi \partial_{x} f=\frac{h-f}{\varepsilon} \\
f(0, x, \xi)=f^{0}(x, \xi)
\end{array}\right.
$$

Furthermore,

$$
f(t, x, \xi)=f^{0}(x-t \xi, \xi) e^{-t / \varepsilon}+\frac{1}{\varepsilon} \int_{0}^{t} e^{-s / \varepsilon} h(t-s, x-s \xi, \xi) d s .
$$


We now prove a stability result for this problem.

Proposition 3.2. - Consider $g, g_{n} \in L^{\infty}(] 0, T\left[, L^{1}(\mathbb{R} \times \mathbb{R})\right)$ such that $g, g_{n} \in D$ a.e.,

$$
\begin{array}{ll}
\iint_{\mathbb{R} \times \mathbb{R}} H(g(t, x, \xi), \xi) d x d \xi \leqslant C_{H}, & \iint_{\mathbb{R} \times \mathbb{R}} g_{0}(t, x, \xi) d x d \xi \leqslant C_{0}, \\
\iint_{\mathbb{R} \times \mathbb{R}} H\left(g_{n}(t, x, \xi), \xi\right) d x d \xi \leqslant C_{H}, & \iint_{\mathbb{R} \times \mathbb{R}}\left(g_{n}\right)_{0}(t, x, \xi) d x d \xi \leqslant C_{0} .
\end{array}
$$

Set

$$
(\rho(t, x), \rho u(t, x))=\int_{\mathbb{R}} g(t, x, \xi) d \xi
$$

and

$$
\left(\rho_{n}(t, x), \rho_{n} u_{n}(t, x)\right)=\int_{\mathbb{R}} g_{n}(t, x, \xi) d \xi .
$$

If $\rho_{n} \underset{n \rightarrow \infty}{\rightarrow} \rho$ and $\rho_{n} u_{n} \underset{n \rightarrow \infty}{\rightarrow} \rho u$ in $L^{1}(] 0, T\left[\times \mathbb{R}_{l o c}\right)$, then there exists a subsequence such that $F\left(g_{n}\right) \underset{n \rightarrow \infty}{\rightarrow} F(g)$ in $C\left([0, T], L^{1}\left(\mathbb{R}_{l o c} \times \mathbb{R}\right)\right)$, where $F(g)$ is the solution to (3.1)-(3.2) with $h=M[g]=M(\rho, u, \xi)$ and $f^{0} \in L^{1}(\mathbb{R} \times$ $\mathbb{R})$ is fixed. Moreover, if $\rho_{n} \rightarrow \rho$ in $L^{1}(] 0, T[\times \mathbb{R})$, then $F\left(g_{n}\right) \rightarrow F(g)$ in $C\left([0, T], L^{1}(\mathbb{R} \times \mathbb{R})\right)$.

Proof. - At first, let us check that $F(g)$ is well defined. Using Proposition 2.5 , we have

$$
\iint_{\mathbb{R} \times \mathbb{R}} H(M[g](t, x, \xi), \xi) d x d \xi \leqslant C_{H}
$$

Besides,

$$
\iint_{\mathbb{R} \times \mathbb{R}} M[g]_{0}(t, x, \xi) d x d \xi=\iint_{\mathbb{R} \times \mathbb{R}} g_{0}(t, x, \xi) d x d \xi \leqslant C_{0} .
$$

Estimate (2.12) applied to $M[g]$ gives then that

$$
\iint_{\mathbb{R} \times \mathbb{R}}\left|M[g]_{1}(t, x, \xi)\right| d x d \xi \leqslant \sqrt{2 C_{0} C_{H}} .
$$

Therefore, we deduce that $M[g] \in L^{\infty}(] 0, T\left[, L^{1}(\mathbb{R} \times \mathbb{R})\right)$, and we have the same result for $g_{n}$ with uniform bounds. 
We turn now to the stability. We can find a subsequence such that $\rho_{n} \rightarrow \rho$ and $\rho_{n} u_{n} \rightarrow \rho u$ a.e. $t, x$. Set $E=\{(t, x) \in[0, T] \times \mathbb{R} ; \rho(t, x)=0\}$. In $E^{c}$, $\rho_{n} \rightarrow \rho$ and $u_{n} \rightarrow u$ a.e. $t, x$, thus $M\left[g_{n}\right] \rightarrow M[g]$ a.e. $(t, x) \in E^{c}, \xi \in \mathbb{R}$. But since

$$
\iint_{(t, x) \in E, x \in B_{R}} \int M\left[g_{n}\right]_{0} d t d x d \xi=\iint_{(t, x) \in E, x \in B_{R}} \rho_{n} d t d x \rightarrow 0,
$$

we deduce with (2.17) and Cauchy-Schwarz inequality that $M\left[g_{n}\right] \rightarrow 0$ in $L^{1}\left((E \times \mathbb{R}) \cap\left\{x \in B_{R}\right\}\right)$. Therefore, after extraction of a subsequence, we finally obtain

$$
\left.M\left[g_{n}\right] \rightarrow M[g] \quad \text { a.e. in }\right] 0, T[\times \mathbb{R} \times \mathbb{R} .
$$

Besides, the estimates of Proposition 2.7 applied to $M\left[g_{n}\right](t, . .$.$) give$ that $M\left[g_{n}\right]$ is weakly compact in $L^{1}(] 0, T\left[\times \mathbb{R}_{l o c} \times \mathbb{R}\right)$. Therefore, we are able to apply the Vitali theorem, which gives that

$$
M\left[g_{n}\right] \rightarrow M[g] \text { in } L^{1}(] 0, T\left[\times \mathbb{R}_{\text {loc }} \times \mathbb{R}\right) .
$$

Now

$$
\begin{aligned}
& \iint_{|x| \leqslant R,|\xi| \leqslant S}\left|\left(F\left(g_{n}\right)-F(g)\right)(t, x, \xi)\right| d x d \xi \\
\leqslant & \frac{1}{\varepsilon} \int_{0}^{t} e^{-s / \varepsilon} \iint_{|x| \leqslant R,|\xi| \leqslant S}\left|\left(M\left[g_{n}\right]-M[g]\right)(t-s, x-s \xi, \xi)\right| d x d \xi d s \\
\leqslant & \frac{1}{\varepsilon}\left\|M\left[g_{n}\right]-M[g]\right\|_{L^{1}(] 0, T\left[\times B_{R+T S} \times B_{S}\right)} \longrightarrow 0,
\end{aligned}
$$

and

$$
\begin{aligned}
& \iint_{|\xi|>S}\left|\left(F\left(g_{n}\right)-F(g)\right)(t, x, \xi)\right| d x d \xi \\
\leqslant & \frac{1}{\varepsilon} \int_{0}^{t} e^{-s / \varepsilon} \iint_{|\xi|>S} \frac{|\xi|}{S}\left|\left(M\left[g_{n}\right]-M[g]\right)(t-s, x-s \xi, \xi)\right| d x d \xi d s \\
\leqslant & \frac{1}{\varepsilon S}\left\|\xi M\left[g_{n}\right]-\xi M[g]\right\|_{L^{1}(] 0, T[\times \mathbb{R} \times \mathbb{R}) .}
\end{aligned}
$$

Since the latter norm is bounded, we deduce easily that $F\left(g_{n}\right) \rightarrow F(g)$ in $C\left([0, T], L^{1}\left(\mathbb{R}_{l o c} \times \mathbb{R}\right)\right)$. 
Finally, if we assume moreover that $\rho_{n} \rightarrow \rho$ in $L^{1}(] 0, T[\times \mathbb{R})$, then

$$
\iiint_{] 0, T[\times \mathbb{R} \times \mathbb{R}} M\left[g_{n}\right]_{0} d t d x d \xi \longrightarrow \iiint_{] 0, T[\times \mathbb{R} \times \mathbb{R}} M[g]_{0} d t d x d \xi,
$$

and since $M\left[g_{n}\right]_{0} \geqslant 0$, we deduce that

$$
\iiint_{|x|>R} M\left[g_{n}\right]_{0} d t d x d \xi \longrightarrow 0 \quad \text { as } R \rightarrow \infty,
$$

uniformly in $n$. With (2.17) and Cauchy-Schwarz inequality, we get also

$$
\iiint_{|x|>R}\left|M\left[g_{n}\right]_{1}\right| d t d x d \xi \rightarrow 0 \quad \text { as } R \rightarrow \infty,
$$

uniformly in $n$, and we conclude that $M\left[g_{n}\right] \rightarrow M[g]$ in $L^{1}(] 0, T[\times \mathbb{R} \times \mathbb{R})$, and therefore $F\left(g_{n}\right) \rightarrow F(g)$ in $C\left([0, T], L^{1}(\mathbb{R} \times \mathbb{R})\right)$.

\section{Existence of a solution}

This section is devoted to the proof of Theorem 1.1. Let $f^{0} \in L^{1}\left(\mathbb{R}_{x} \times \mathbb{R}_{\xi}\right)$ satisfy the assumptions of Theorem 1.1, namely

$$
\begin{gathered}
f^{0}(x, \xi) \in D \text { a.e. in } \mathbb{R} \times \mathbb{R}, \\
\iint_{\mathbb{R} \times \mathbb{R}} H\left(f^{0}(x, \xi), \xi\right) d x d \xi=C_{H}^{0}<\infty, \\
\iint_{\mathbb{R} \times \mathbb{R}} x^{2}\left(f^{0}\right)_{0}(x, \xi) d x d \xi=C_{\Delta}^{0}<\infty,
\end{gathered}
$$

and set

$$
\iint_{\mathbb{R} \times \mathbb{R}}\left(f^{0}\right)_{0}(x, \xi) d x d \xi=C_{0}
$$

We take $C_{H}$ and $C_{\Delta}$ as

$$
C_{H}=C_{H}^{0}, \quad C_{\Delta}=\max \left(C_{\Delta}^{0} ; \frac{4 \varepsilon^{2}}{\theta} C_{H}^{0}\right) .
$$


Define $C$ to be the set of all $g \in L^{\infty}(] 0, T\left[, L^{1}(\mathbb{R} \times \mathbb{R})\right)$ satisfying $(C 1)-$ $(C 4)$ for a.e. $t \in[0, T]$, where

$$
\begin{aligned}
& (C 1) \quad g(t, x, \xi) \in D \quad \text { a.e. in } \mathbb{R} \times \mathbb{R}, \\
& (C 2) \quad \iint_{\mathbb{R} \times \mathbb{R}} H(g(t, x, \xi), \xi) d x d \xi \leqslant C_{H}, \\
& \text { (C3) } \iint_{\mathbb{R} \times \mathbb{R}} g_{0}(t, x, \xi) d x d \xi \leqslant C_{0}, \\
& \text { (C4) } \iint_{\mathbb{R} \times \mathbb{R}} x^{2} g_{0}(t, x, \xi) d x d \xi \leqslant C_{\Delta}(1+t / \varepsilon)^{2} .
\end{aligned}
$$

Let us also introduce

$$
\begin{array}{r}
\tilde{C}=\left\{g \in C\left([0, T], L^{1}(\mathbb{R} \times \mathbb{R})\right) \text { satisfying }(C 5),\right. \\
\text { and }(C 1)-(C 4) \text { for all } t \in[0, T]\},
\end{array}
$$

with

$$
\text { (C5) } \partial_{t} g+\xi \partial_{x} g+\frac{g}{\varepsilon} \in \frac{C}{\varepsilon} \text {. }
$$

The initial data $f^{0}$ being fixed, for any $g \in C$, we denote by $F(g)$ the solution to (3.1)-(3.2) with $h=M[g]$.

Lemma 4.1. - If $g \in C$, then $M[g] \in C$ and $F(g) \in \tilde{C}$.

Proof. - Let $g \in C$. As in the proof of Proposition 3.2, $M[g] \in L^{\infty}(] 0, T[$, $\left.L^{1}(\mathbb{R} \times \mathbb{R})\right)$ and we get easily that $M[g] \in C$. Next, according to Lemma 3.1, $F(g) \in C\left([0, T], L^{1}(\mathbb{R} \times \mathbb{R})\right)$, and in order to prove that $F(g) \in \tilde{C}$, we need to prove $(C 1)-(C 5)$. Condition $(C 5)$ is satisfied since $M[g] \in C$. Then, for $i=0 ; 1$, we have

$$
F(g)_{i}(t, x, \xi)=\left(f^{0}\right)_{i}(x-t \xi, \xi) e^{-t / \varepsilon}+\frac{1}{\varepsilon} \int_{0}^{t} e^{-s / \varepsilon} M[g]_{i}(t-s, x-s \xi, \xi) d s .
$$

Obviously, $F(g)_{0} \geqslant 0$. Furthermore, if $F(g)_{0}(t, x, \xi)=0$ then $\left(f^{0}\right)_{0}(x-$ $t \xi, \xi)=0$ and $\int_{0}^{t} e^{-s / \varepsilon} M[g]_{0}(t-s, x-s \xi, \xi) d s=0$, thus $M[g]_{0}(t-s, x-$ $s \xi, \xi)=0$ a.e. $s \in] 0, t\left[\right.$. But $f^{0} \in D$ and $M[g] \in D$, thus $\left(f^{0}\right)_{1}(x-t \xi, \xi)=0$ and $M[g]_{1}(t-s, x-s \xi, \xi)=0$ a.e. $\left.s \in\right] 0, t\left[\right.$, and therefore $F(g)_{1}(t, x, \xi)=0$. 
Hence $F[g] \in D \forall t \in[0, T]$, a.e. $x, \xi$, and $(C 1)$ is satisfied. Next, if $t>0$, we can write

$$
F(g)(t, x, \xi)=f^{0}(x-t \xi, \xi) e^{-t / \varepsilon}+\left(1-e^{-t / \varepsilon}\right) \int_{0}^{t} M[g](t-s, x-s \xi, \xi) \frac{e^{-s / \varepsilon} d s}{\int_{0}^{t} e^{-\sigma / \varepsilon} d \sigma}
$$

and using Jensen's inequality with the convex function $H$, we get

$$
\begin{aligned}
& H(F(g)(t, x, \xi), \xi) \\
\leqslant & H\left(f^{0}(x-t \xi, \xi), \xi\right) e^{-t / \varepsilon} \\
& +\left(1-e^{-t / \varepsilon}\right) H\left(\int_{0}^{t} M[g](t-s, x-s \xi, \xi) \frac{e^{-s / \varepsilon} d s}{\int_{0}^{t} e^{-\sigma / \varepsilon} d \sigma}, \xi\right) \\
\leqslant & H\left(f^{0}(x-t \xi, \xi), \xi\right) e^{-t / \varepsilon} \\
& +\left(1-e^{-t / \varepsilon}\right) \int_{0}^{t} H(M[g](t-s, x-s \xi, \xi), \xi) \frac{e^{-s / \varepsilon} d s}{\int_{0}^{t} e^{-\sigma / \varepsilon} d \sigma} \\
= & H\left(f^{0}(x-t \xi, \xi), \xi\right) e^{-t / \varepsilon} \\
& +\frac{1}{\varepsilon} \int_{0}^{t} e^{-s / \varepsilon} H(M[g](t-s, x-s \xi, \xi), \xi) d s,
\end{aligned}
$$

and thus

$$
\iint_{\mathbb{R} \times \mathbb{R}} H(F(g)(t, x, \xi), \xi) d x d \xi \leqslant e^{-t / \varepsilon} C_{H}^{0}+\left(1-e^{-t / \varepsilon}\right) C_{H} \leqslant C_{H},
$$

and $(C 2)$ is satisfied. Similarly, integrating (4.8) with $i=0$ with respect to $(x, \xi)$ gives $(C 3)$. It only remains now to prove $(C 4)$. We have by $(2.13)$

$$
\begin{aligned}
& \iint_{\mathbb{R} \times \mathbb{R}} x^{2}\left(f^{0}\right)_{0}(x-t \xi, \xi) d x d \xi \\
= & \iint(y+t \xi)^{2}\left(f^{0}\right)_{0}(y, \xi) d y d \xi \\
\leqslant & \left(\sqrt{\iint y^{2}\left(f^{0}\right)_{0}(y, \xi) d y d \xi}+t \sqrt{\iint \xi^{2}\left(f^{0}\right)_{0}(y, \xi) d y d \xi}\right)^{2} \\
\leqslant & \left(\sqrt{C_{\Delta}^{0}}+t \sqrt{4 C_{H}^{0} / \theta}\right)^{2} .
\end{aligned}
$$

Then, since $M[g] \in C$, relation $(C 4)$ gives

$$
\iint_{\mathbb{R} \times \mathbb{R}} x^{2} M[g]_{0}(s, x, \xi) d x d \xi \leqslant C_{\Delta}(1+s / \varepsilon)^{2},
$$


Florent Berthelin, François Bouchut

hence

$$
\begin{aligned}
& \iint_{\mathbb{R} \times \mathbb{R}} x^{2} M[g]_{0}(t-s, x-s \xi, \xi) d x d \xi \\
= & \iint(y+s \xi)^{2} M[g]_{0}(t-s, y, \xi) d y d \xi \\
\leqslant & \left(\sqrt{\iint y^{2} M[g]_{0}(t-s, y, \xi) d y d \xi}+s \sqrt{\iint \xi^{2} M[g]_{0}(t-s, y, \xi) d y d \xi}\right)^{2} \\
\leqslant & \left(\sqrt{C_{\Delta}\left(1+\frac{t-s}{\varepsilon}\right)^{2}}+s \sqrt{4 C_{H} / \theta}\right)^{2}
\end{aligned}
$$

Therefore, with (4.5),

$$
\begin{aligned}
& \iint_{\mathbb{R} \times \mathbb{R}} x^{2} F(g)_{0}(t, x, \xi) d x d \xi \\
\leqslant & \left(\sqrt{C_{\Delta}}+t \sqrt{4 C_{H} / \theta}\right)^{2} e^{-t / \varepsilon} \\
& +\frac{1}{\varepsilon} \int_{0}^{t} e^{-s / \varepsilon}\left(\sqrt{C_{\Delta}}\left(1+\frac{t-s}{\varepsilon}\right)+s \sqrt{4 C_{H} / \theta}\right)^{2} d s \\
\leqslant & C_{\Delta}\left(1+\frac{t}{\varepsilon}\right)^{2} e^{-t / \varepsilon} \\
& +\frac{1}{\varepsilon} \int_{0}^{t} e^{-s / \varepsilon} C_{\Delta}\left(1+\frac{t}{\varepsilon}\right)^{2} d s \\
= & C_{\Delta}\left(1+\frac{t}{\varepsilon}\right)^{2} .
\end{aligned}
$$

This ends the proof of the lemma.

Since $\tilde{C} \subset C$, we are now able to define $F: \tilde{C} \rightarrow \tilde{C}$ and we shall use the Schauder theorem to prove Theorem 1.1.

LemMA 4.2. - The sets $C$ and $\tilde{C}$ are convex and not empty, $C$ is compact for the weak topology of $L^{1}(] 0, T[\times \mathbb{R} \times \mathbb{R})$, and $\tilde{C}$ is closed in $C\left([0, T], L^{1}(\mathbb{R} \times \mathbb{R})\right)$.

Proof. - Since $H$ is convex, it is obvious that $C$ and $\tilde{C}$ are convex. Then, the constant $f^{0}$ belongs to $C$, and by Lemma $4.1, F\left(f_{0}\right) \in \tilde{C}$. Thus $C$ and $\tilde{C}$ are not empty. Next, from Proposition 2.7, (C4), (2.17) and Dunford-Pettis' 
theorem, $C$ is relatively weakly compact in $L^{1}(] 0, T[\times \mathbb{R} \times \mathbb{R})$. Let us prove now that $C$ is closed in weak $L^{1}(] 0, T[\times \mathbb{R} \times \mathbb{R})$. Since $C$ is convex, it is enough to prove that $C$ is closed in strong $L^{1}(] 0, T[\times \mathbb{R} \times \mathbb{R})$. Thus let $\left(g_{n}\right)_{n}$ be a sequence in $C$ which converges to $g$ in $L^{1}(] 0, T[\times \mathbb{R} \times \mathbb{R})$. After extraction of a subsequence, $g_{n}(t,.) \rightarrow g(t,$.$) in L^{1}(\mathbb{R} \times \mathbb{R})$ and a.e. for a.e. $\left.t \in\right] 0, T$. First, $\left(g_{n}\right)_{0} \geqslant 0$ thus $g_{0} \geqslant 0$. Then, since by $(2.17)\left|\left(g_{n}\right)_{1}\right| \leqslant \sqrt{2 H\left(g_{n}, \xi\right)\left(g_{n}\right)_{0}}$, we get for a fixed time $t$ and any Borel set $\mathcal{V}$ by Fatou's lemma and CauchySchwarz inequality

$$
\begin{aligned}
\iint_{\mathcal{V}}\left|g_{1}(t, x, \xi)\right| d x d \xi & \leqslant \underline{\lim } \iint_{\mathcal{V}} \sqrt{2 H\left(g_{n}, \xi\right)\left(g_{n}\right)_{0}} d x d \xi \\
& \leqslant \underline{\lim } \sqrt{2 C_{H} \iint_{\mathcal{V}}\left(g_{n}\right)_{0} d x d \xi} \\
& =\sqrt{2 C_{H} \iint_{\mathcal{V}} g_{0} d x d \xi .}
\end{aligned}
$$

Taking $\mathcal{V}=\left\{x, \xi ; g_{0}(t, x, \xi)=0\right\}$, we obtain that $g_{1}(t, x, \xi)$ vanishes a.e. in $\mathcal{V}$, thus $g(t, x, \xi) \in D$ a.e. Then, we apply Fatou's lemma again, and get

$$
\begin{aligned}
\iint_{\mathbb{R} \times \mathbb{R}} H(g(t, x, \xi), \xi) d x d \xi & =\iint_{g_{0}>0} H(g(t, x, \xi), \xi) d x d \xi \\
& \leqslant \underline{\lim } \iint_{g_{0}>0} H\left(g_{n}(t, x, \xi), \xi\right) d x d \xi \\
& \leqslant C_{H} .
\end{aligned}
$$

Similar applications of Fatou's lemma finally give that $g \in L^{\infty}(] 0, T$, $\left.L^{1}(\mathbb{R} \times \mathbb{R})\right)$, and $g \in C$. The closedness of $\tilde{C}$ in $C\left([0, T], L^{1}(\mathbb{R} \times \mathbb{R})\right)$ is treated in a similar way, and (C5) follows from the compactness of $C$.

Lemma 4.3. - $F$ is continuous in $\tilde{C}$ for the topology $C([0, T]$, $\left.L^{1}(\mathbb{R} \times \mathbb{R})\right)$.

Proof. - Let $g_{n}, g \in \tilde{C}$ with $g_{n} \rightarrow g$ in $C\left([0, T], L^{1}(\mathbb{R} \times \mathbb{R})\right)$. Then, with the notations of Proposition 3.2, $\rho_{n} \rightarrow \rho$ and $\rho_{n} u_{n} \rightarrow \rho u$ in $C\left([0, T], L^{1}(\mathbb{R})\right)$. Thus Proposition 3.2 gives the existence of a subsequence such that $F\left(g_{n}\right) \rightarrow F(g)$ in $C\left([0, T], L^{1}(\mathbb{R} \times \mathbb{R})\right)$, which is enough to prove the continuity of $F$.

Lemma 4.4. - F( $\tilde{C})$ is relatively compact in $C\left([0, T], L^{1}(\mathbb{R} \times \mathbb{R})\right)$. 
Proof. - Let $f_{n}=F\left(g_{n}\right), g_{n} \in \tilde{C}$ be a sequence in $F(\tilde{C})$. Since $\tilde{C} \subset C$, by Lemma 4.2 there exist $g \in C$ and a subsequence such that $g_{n} \rightarrow g$ in weak $L^{1}(] 0, T[\times \mathbb{R} \times \mathbb{R})$. Then with the notations of Proposition 3.2, $\rho_{n} \rightarrow \rho$, $\rho_{n} u_{n} \rightarrow \rho u$ in weak $L^{1}(] 0, T[\times \mathbb{R})$. But by $(C 5), \partial_{t} g_{n}+\xi \partial_{x} g_{n}+g_{n} / \varepsilon=$ $h_{n} / \varepsilon$, with $h_{n} \in C$. By the compactness averaging lemma of [8] and by (2.13)-(2.14), we deduce that $\int_{\mathbb{R}} g_{n}(t, x, \xi) d \xi$ is compact in $L_{\text {loc }}^{1}(] 0, T[\times \mathbb{R})$. We conclude that $\rho_{n} \rightarrow \rho$ and $\rho_{n} u_{n} \rightarrow \rho u$ in $L^{1}(] 0, T[\times \mathbb{R})$. Proposition 3.2 gives thus the existence of a subsequence such that $f_{n}=F\left(g_{n}\right) \rightarrow F(g)$ in $C\left([0, T], L^{1}(\mathbb{R} \times \mathbb{R})\right)$.

Proof of Theorem 1.1. - We apply Schauder's theorem in $C\left([0, T], L^{1}(\mathbb{R} \times\right.$ $\mathbb{R})$ ) to the operator $F: \tilde{C} \rightarrow \tilde{C}$. Using all the results of this section, $\tilde{C}$ is convex, closed and non empty, $F$ is continuous $\tilde{C} \rightarrow \tilde{C}$ and $F(\tilde{C})$ is relatively compact. Thus we conclude the existence of a fixed point $f \in \tilde{C}$ verifying $F(f)=f$. This gives a solution in $[0, T]$ for any $T>0$, and by extraction of a diagonal subsequence, we obtain a solution in $[0, \infty[$. Relation (1.21) comes clearly from (1.2) because $\xi f(t, x, \xi) \in L^{\infty}(] 0, \infty\left[, L^{1}(\mathbb{R} \times \mathbb{R})\right)$ by Proposition 2.7, and (1.22) is obtained by integration of (4.8).

Proof of Theorem 1.2. - If the assumption (1.17) is not satisfied, we have to use the Tychonoff-Schauder fixed point theorem in the locally convex topological vector space $C\left([0, T], L^{1}\left(\mathbb{R}_{l o c, x} \times \mathbb{R}_{\xi}\right)\right)$. Let us mention the changes that need to be performed to the results of Section 4 . First, we have to remove condition $(C 4)$ in the definitions of $C$ and $\tilde{C}$. Then, in Lemma $4.2, C$ is compact for the weak topology of $L^{1}(] 0, T\left[\times \mathbb{R}_{l o c} \times \mathbb{R}\right)$, and $\tilde{C}$ is closed in $C\left([0, T], L^{1}\left(\mathbb{R}_{l o c} \times \mathbb{R}\right)\right.$. In Lemma $4.3, F$ is continuous in $\tilde{C}$ for the topology $C\left([0, T], L^{1}\left(\mathbb{R}_{l o c} \times \mathbb{R}\right)\right)$, and in Lemma $4.4, F(\tilde{C})$ is relatively compact in $C\left([0, T], L^{1}\left(\mathbb{R}_{l o c} \times \mathbb{R}\right)\right)$.

\section{Entropy equation}

In this section we prove that the entropy $H(f(t, x, \xi), \xi)$ satisfies a renormalized equation, as stated in Theorem 1.3. The difficulty is that $H$ is not smooth at the origin, and we have to build a monotone approximation of it that is convex, smooth and that has slow growth at infinity.

\subsection{Approximation of $H$}

The most singular term in $H$ in (1.13) is obviously

$$
\Phi(f)=\mathbf{I}_{f_{0}>0} f_{1}^{2} / f_{0}, \quad f \in D .
$$


We begin by translating the singularity $f_{0}=0$ to $f_{0}=-\delta$, by defining for $\delta>0$

$$
\Phi_{\delta}(f)=\frac{f_{1}^{2}}{f_{0}+\delta}, \quad f \in D,
$$

which is convex and smooth in $D$, hence

$$
\Phi_{\delta}(f)=\sup _{g \in D}\left(\Phi_{\delta}(g)+\Phi_{\delta}^{\prime}(g) \cdot(f-g)\right), \quad f \in D .
$$

In order to obtain a linear growth at infinity, we set

$$
\begin{gathered}
\varphi_{\delta}(f)=\sup _{g \in D_{\delta}}\left(\Phi_{\delta}(g)+\Phi_{\delta}^{\prime}(g) \cdot(f-g)\right), \quad f \in D, \\
D_{\delta}=\left\{f \in D ;\left|\frac{f_{1}}{f_{0}+\delta}\right| \leqslant c_{\delta}\right\}, \quad c_{\delta}=1 / \delta^{2} .
\end{gathered}
$$

One can check that with this choice of $c_{\delta}$,

$$
\text { if } 0<\delta^{\prime} \leqslant \delta \text { then } D_{\delta} \subset D_{\delta^{\prime}} .
$$

We have obviously $\varphi_{\delta}(f) \leqslant \Phi_{\delta}(f) \leqslant \Phi(f)$.

Lemma 5.1. - i) The value of $\varphi_{\delta}$ is

$$
\varphi_{\delta}(f)= \begin{cases}\frac{f_{1}^{2}}{f_{0}+\delta} & \text { if } f \in D_{\delta}, \\ -c_{\delta}^{2}\left(f_{0}+\delta\right)+2 c_{\delta}\left|f_{1}\right| & \text { if } f \in D \backslash D_{\delta},\end{cases}
$$

ii) the function $\varphi_{\delta}$ is convex in $D$, nonnegative, $\varphi_{\delta}(0)=0$,

iii) we have $\varphi_{\delta}(f) \leqslant 2 c_{\delta}\left|f_{1}\right|$ for $f \in D$, $\delta \rightarrow 0$,

$i v)$ if $0<\delta^{\prime} \leqslant \delta$ then $\varphi_{\delta}(f) \leqslant \varphi_{\delta^{\prime}}(f)$ for $f \in D$, and $\varphi_{\delta}(f) \uparrow \Phi(f)$ as

$v)$ the function $\varphi_{\delta}$ is $C^{1}$ in $\bar{D}$ and $\varphi_{\delta}^{\prime}$ is bounded in $\bar{D}$.

Preuve. - i) If $f \in D_{\delta}$, obviously $\varphi_{\delta}(f)=\Phi_{\delta}(f)$. If $f \in D \backslash D_{\delta}$,

$$
\Phi_{\delta}(g)+\Phi_{\delta}^{\prime}(g) \cdot(f-g)=-\frac{g_{1}^{2}}{\left(g_{0}+\delta\right)^{2}}\left(f_{0}+\delta\right)+2 \frac{g_{1}}{g_{0}+\delta} f_{1},
$$

hence

$$
\begin{gathered}
\varphi_{\delta}(f)=\sup _{-c_{\delta} \leqslant x \leqslant c_{\delta}}\left(-x^{2}\left(f_{0}+\delta\right)+2 x f_{1}\right) . \\
-623-
\end{gathered}
$$


If $f_{1} \geqslant 0$, the supremum is reached at $x=c_{\delta}$ because $c_{\delta}<f_{1} /\left(f_{0}+\delta\right)$, and if $f_{1} \leqslant 0$, it is reached at $-c_{\delta}$, and this gives the result.

ii) The convexity is obvious from the definition, the nonnegativity also since $0 \in D_{\delta}$, and $\varphi_{\delta}(0)$ is computed by (5.6).

iii) If $f \in D_{\delta}$ then $\varphi_{\delta}(f)=f_{1}^{2} /\left(f_{0}+\delta\right) \leqslant c_{\delta}\left|f_{1}\right|$, and if $f \in D \backslash D_{\delta}$, then $\varphi_{\delta}(f)=-c_{\delta}^{2}\left(f_{0}+\delta\right)+2 c_{\delta}\left|f_{1}\right| \leqslant 2 c_{\delta}\left|f_{1}\right|$.

$i v)$ Let $0<\delta^{\prime} \leqslant \delta$. If $f \in D_{\delta}$ then $f \in D_{\delta^{\prime}}$ by (5.5), thus $\varphi_{\delta}(f)=$ $f_{1}^{2} /\left(f_{0}+\delta\right) \leqslant f_{1}^{2} /\left(f_{0}+\delta^{\prime}\right)=\varphi_{\delta^{\prime}}(f)$. If $f \in D_{\delta^{\prime}} \backslash D_{\delta}$, then $\varphi_{\delta}(f) \leqslant \Phi_{\delta}(f) \leqslant$ $\Phi_{\delta^{\prime}}(f)=\varphi_{\delta^{\prime}}(f)$. If $f \in D \backslash D_{\delta^{\prime}}$, i.e. $\left|f_{1}\right|>c_{\delta^{\prime}}\left(f_{0}+\delta^{\prime}\right)$, then since $c_{\delta} \leqslant c_{\delta^{\prime}}$,

$$
\begin{aligned}
\varphi_{\delta}(f)-\varphi_{\delta^{\prime}}(f) & =-c_{\delta}^{2}\left(f_{0}+\delta\right)+c_{\delta^{\prime}}^{2}\left(f_{0}+\delta^{\prime}\right)+2\left(c_{\delta}-c_{\delta^{\prime}}\right)\left|f_{1}\right| \\
& \leqslant-c_{\delta}^{2}\left(f_{0}+\delta^{\prime}\right)+c_{\delta^{\prime}}^{2}\left(f_{0}+\delta^{\prime}\right)+2\left(c_{\delta}-c_{\delta^{\prime}}\right) c_{\delta^{\prime}}\left(f_{0}+\delta^{\prime}\right) \\
& =-\left(c_{\delta^{\prime}}-c_{\delta}\right)^{2}\left(f_{0}+\delta^{\prime}\right) \\
& \leqslant 0 .
\end{aligned}
$$

Finally, if $f \in D$ is fixed, for small enough $\delta$ we have $f \in D_{\delta}, \varphi_{\delta}(f)=$ $f_{1}^{2} /\left(f_{0}+\delta\right) \rightarrow \mathbf{I}_{f_{0}>0} f_{1}^{2} / f_{0}$ as $\delta \rightarrow 0$.

$v$ ) Formula (5.6) gives obviously two smooth functions. We have to check the continuity of the functions and their derivatives at the frontier where $\left|f_{1}\right|=c_{\delta}\left(f_{0}+\delta\right)$. The continuity of $\varphi_{\delta}$ is straightforward. For the derivatives, we have

$$
\varphi_{\delta}^{\prime}(f)=\left\{\begin{array}{l}
\left(\frac{-f_{1}^{2}}{\left(f_{0}+\delta\right)^{2}}, \frac{2 f_{1}}{f_{0}+\delta}\right), \quad f \in \operatorname{int}\left(D_{\delta}\right), \\
\left(-c_{\delta}^{2}, 2 c_{\delta} \operatorname{sgn} f_{1}\right), \quad f \in \operatorname{int}\left(D \backslash D_{\delta}\right),
\end{array}\right.
$$

and we see that the two formula match when $\left|f_{1}\right|=c_{\delta}\left(f_{0}+\delta\right)$. We finally notice that $\left|\varphi_{\delta}^{\prime}\right| \leqslant c_{\delta}^{2}+2 c_{\delta}$.

\subsection{Sketch of the proof}

In order to prove the equation on $H(f(t, x, \xi), \xi)$, we decompose $H$ as

$$
H(f, \xi)=\frac{\theta}{1-\theta} \frac{\xi^{2}}{2} f_{0}-\frac{\theta}{1-\theta} \xi f_{1}+\frac{\theta}{2 c_{\gamma, \kappa}^{1 / \lambda}} \frac{f_{0}^{1+1 / \lambda}}{1+1 / \lambda}+\frac{1}{1-\theta} \frac{1}{2} \mathbf{I}_{f_{0}>0} \frac{f_{1}^{2}}{f_{0}},
$$

and we are going to consider each term successively. A crucial argument is the following lemma.

Lemma 5.2. - Assume that $g \in C\left([0, T], L_{\text {loc }}^{1}\left(\mathbb{R}_{x} \times \mathbb{R}_{\xi}\right)\right)$ verifies

$$
g(0) \in L^{1}(\mathbb{R} \times \mathbb{R}), \quad \text { and } \quad \partial_{t} g+\xi \partial_{x} g=h \in L^{1}(] 0, T[\times \mathbb{R} \times \mathbb{R}) .
$$


BGK system for gas dynamics

Then

$$
\begin{gathered}
g \in C\left([0, T], L^{1}(\mathbb{R} \times \mathbb{R})\right), \\
\xi g \in L^{1}(] 0, T\left[\times B_{R} \times \mathbb{R}\right), \quad R>0, \\
g(t, x, \xi)=g(0, x-t \xi, \xi)+\int_{0}^{t} h(t-s, x-s \xi, \xi) d s, \\
\left.\partial_{t} \int g d \xi+\partial_{x} \int \xi g d \xi=\int h d \xi \quad \text { in } \quad\right] 0, T[\times \mathbb{R}, \\
\left.\frac{d}{d t} \iint_{\mathbb{R} \times \mathbb{R}} g(t, x, \xi) d x d \xi=\iint_{\mathbb{R} \times \mathbb{R}} h(t, x, \xi) d x d \xi \quad \text { in } \quad\right] 0, T[.
\end{gathered}
$$

Proof. - Define $\tilde{g}(t, x, \xi)=g(t, x+t \xi, \xi)$. Then $\tilde{g} \in C\left([0, T], L_{l o c}^{1}(\mathbb{R} \times\right.$ $\mathbb{R})$ ), and $\tilde{g}$ verifies $\tilde{g}(0)=g(0) \in L^{1}(\mathbb{R} \times \mathbb{R}), \partial_{t} \tilde{g}=h(t, x+t \xi, \xi) \in$ $L^{1}(] 0, T[\times \mathbb{R} \times \mathbb{R})$. Therefore,

$$
\tilde{g}(t, x, \xi)=g(0, x, \xi)+\int_{0}^{t} h(s, x+s \xi, \xi) d s,
$$

and we deduce (5.12) and (5.10). Then, (5.11) comes from the higher moment lemma of [15], and (5.13) follows by integration of (5.9). Finally, integrating (5.12) with respect to $(x, \xi)$, we get for any $t \in[0, T]$

$$
\iint g(t, x, \xi) d x d \xi=\iint g(0, x, \xi) d x d \xi+\int_{0}^{t} \iint_{\mathbb{R} \times \mathbb{R}} h(s, x, \xi) d x d \xi d s,
$$

which is the integral form of (5.14).

\subsection{Linear terms}

We are now able to treat the first two terms in (5.8). From now on, $f$ denotes the solution obtained in Theorems 1.1 or 1.2 . We recall that the estimates of Proposition 2.7 apply to $f(t,$.$) and to M[f](t,$.$) for any$ $t \geqslant 0$. Thus we can apply Lemma 5.2 to $\xi^{2} f_{0}(t, x, \xi)$ and $\xi f_{1}(t, x, \xi)$, and we conclude that

$$
\xi^{2} f_{0}(t, x, \xi), \quad \xi f_{1}(t, x, \xi) \quad \in C\left(\left[0, \infty\left[, L^{1}(\mathbb{R} \times \mathbb{R})\right),\right.\right.
$$

and all the conclusions of Lemma 5.2 are valid. 


\subsection{Nonlinear terms}

Let us first consider the last term in (5.8), corresponding to $\Phi(f)=$ $\mathbf{I}_{f_{0}>0} f_{1}^{2} / f_{0}$. We use the approximation $\varphi_{\delta}(f)$ defined in Section 5.1. Since $\varphi_{\delta}$ is $C^{1}$ and globally Lipschitz continuous, a vector adaptation of the classical renormalization property allows to multiply $(1.2)$ by $\varphi_{\delta}^{\prime}(f)$, which yields

$$
\partial_{t} \varphi_{\delta}(f)+\xi \partial_{x} \varphi_{\delta}(f)=\varphi_{\delta}^{\prime}(f) \frac{M[f]-f}{\varepsilon} .
$$

Since $\varphi_{\delta}(f) \in C\left([0, T], L^{1}(\mathbb{R} \times \mathbb{R})\right)$, we can apply Lemma 5.2 to $\varphi_{\delta}(f)$. We wish now to let $\delta \rightarrow 0$ in (5.17). We notice that $\Phi$ in (5.1) is smooth outside the origin, and we define $\Phi^{\prime}(0)=0$ by convention. Thus

$$
\Phi^{\prime}(f)=\left(-\mathbf{I}_{f_{0}>0} \frac{f_{1}^{2}}{f_{0}^{2}}, \mathbf{I}_{f_{0}>0} \frac{2 f_{1}}{f_{0}}\right), \quad f \in D .
$$

Lemma 5.3. - The terms $\partial_{0} \Phi(f) f_{0}, \partial_{1} \Phi(f) f_{1}, \partial_{0} \Phi(f) M_{0}[f], \partial_{1} \Phi(f)$ $M_{1}[f]$ lie in $L^{1}(] 0, T[\times \mathbb{R} \times \mathbb{R})$. The right-hand side of (5.17) is bounded in $L^{1}(] 0, T[\times \mathbb{R} \times \mathbb{R})$ uniformly in $\delta$, and tends to $\Phi^{\prime}(f)(M[f]-f) / \varepsilon$ in $L^{1}(] 0, T[\times \mathbb{R} \times \mathbb{R})$ as $\delta \rightarrow 0$.

Proof. - Since $0 \leqslant \Phi(f) \leqslant 2 H(f, \xi)$ for any $\xi$, we have

$$
\Phi(f(t, x, \xi)), \quad \Phi(M[f](t, x, \xi)) \in L^{\infty}(] 0, \infty\left[, L^{1}(\mathbb{R} \times \mathbb{R})\right) .
$$

Then, $\partial_{0} \Phi(f) f_{0}=-\mathbf{I}_{f_{0}>0} f_{1}^{2} / f_{0}=-\Phi(f) \in L^{\infty}(] 0, \infty\left[, L^{1}(\mathbb{R} \times \mathbb{R})\right)$, $\partial_{1} \Phi(f) f_{1}=\mathbf{1}_{f_{0}>0} 2 f_{1}^{2} / f_{0}=2 \Phi(f) \in L^{\infty}(] 0, \infty\left[, L^{1}(\mathbb{R} \times \mathbb{R})\right)$. Next, since $0 \leqslant \varphi_{\delta}(f) \leqslant \Phi(f), \varphi_{\delta}(f)$ is bounded in $C\left([0, T], L^{1}(\mathbb{R} \times \mathbb{R})\right)$ independently of $\delta$. Therefore, by (5.17) and by applying the identity (5.15) to $\varphi_{\delta}(f)$, we obtain that

$$
\iiint_{0, T \times \mathbb{R} \times \mathbb{R}} \varphi_{\delta}^{\prime}(f)(M[f]-f) d t d x d \xi \quad \text { is bounded independently of } T \text { and } \delta .
$$

Then, since $\varphi_{\delta}$ is convex in $D$,

$$
\varphi_{\delta}(M[f])-\varphi_{\delta}(f)-\varphi_{\delta}^{\prime}(f)(M[f]-f) \geqslant 0 .
$$

According to (5.20), this function is bounded in $L^{1}(] 0, T[\times \mathbb{R} \times \mathbb{R})$ independently of $\delta$. Observing that with (5.7), $\varphi_{\delta}^{\prime}(f) \rightarrow \Phi^{\prime}(f)$ as $\delta \rightarrow 0$, by applying Fatou's lemma, we deduce that

$$
\Phi(M[f])-\Phi(f)-\Phi^{\prime}(f)(M[f]-f) \quad \in L^{1}(] 0, T[\times \mathbb{R} \times \mathbb{R}),
$$


and therefore

$$
\Phi^{\prime}(f) \cdot M[f]=-\mathbf{I}_{f_{0}>0} \frac{f_{1}^{2}}{f_{0}^{2}} M_{0}[f]+\mathbf{I}_{f_{0}>0} \frac{2 f_{1}}{f_{0}} M_{1}[f] \in L^{1}(] 0, T[\times \mathbb{R} \times \mathbb{R}) .
$$

From the inequality

$$
2\left|\frac{f_{1}}{f_{0}} M_{1}[f]\right| \leqslant \frac{1}{4} \frac{f_{1}^{2}}{f_{0}^{2}} M_{0}[f]+4 \frac{M_{1}[f]^{2}}{M_{0}[f]} \mathbf{I}_{M_{0}[f]>0},
$$

we deduce

$$
\begin{aligned}
\frac{f_{1}^{2}}{f_{0}^{2}} M_{0}[f] & =2 \frac{f_{1}}{f_{0}} M_{1}[f]-\left(-\frac{f_{1}^{2}}{f_{0}^{2}} M_{0}[f]+2 \frac{f_{1}}{f_{0}} M_{1}[f]\right) \\
& \leqslant \frac{1}{4} \frac{f_{1}^{2}}{f_{0}^{2}} M_{0}[f]+4 \frac{M_{1}[f]^{2}}{M_{0}[f]} \mathbf{I}_{M_{0}[f]>0}-\left(-\frac{f_{1}^{2}}{f_{0}^{2}} M_{0}[f]+2 \frac{f_{1}}{f_{0}} M_{1}[f]\right),
\end{aligned}
$$

and thus

$$
0 \leqslant \mathbf{I}_{f_{0}>0} \frac{3}{4} \frac{f_{1}^{2}}{f_{0}^{2}} M_{0}[f] \leqslant 4 \Phi(M[f])-\Phi^{\prime}(f) \cdot M[f] .
$$

We conclude that

$$
\partial_{0} \Phi(f) M_{0}[f], \quad \partial_{1} \Phi(f) M_{1}[f] \quad \in L^{1}(] 0, T[\times \mathbb{R} \times \mathbb{R}) .
$$

Finally, we observe with (5.7) that

$$
\left|\partial_{0} \varphi_{\delta}(f)\right| \leqslant\left|\partial_{0} \Phi(f)\right|, \quad\left|\partial_{1} \varphi_{\delta}(f)\right| \leqslant\left|\partial_{1} \Phi(f)\right|,
$$

and we conclude with Lebesgue's theorem the convergence of each term of the right-hand side of (5.17) in $L^{1}(] 0, T[\times \mathbb{R} \times \mathbb{R})$ as $\delta \rightarrow 0$.

Now, we are able to conclude. For any $t \geqslant 0, \varphi_{\delta}(f(t,).) \rightarrow \Phi(f(t,)$.$) in$ $L^{1}(\mathbb{R} \times \mathbb{R})$ as $\delta \rightarrow 0$ by Lebesgue's theorem. But we can write (5.12) for $\varphi_{\delta}(f)$,

$$
\varphi_{\delta}(f(t, x, \xi))=\varphi_{\delta}\left(f^{0}(x-t \xi, \xi)\right)+\int_{0}^{t} \varphi_{\delta}^{\prime}(f) \frac{M[f]-f}{\varepsilon}(t-s, x-s \xi, \xi) d s .
$$

By Lemma 5.3, we can pass to the limit in the right-hand side in $C([0, T]$, $\left.L^{1}(\mathbb{R} \times \mathbb{R})\right)$. Therefore,

$$
\begin{gathered}
\Phi(f(t, x, \xi))=\Phi\left(f^{0}(x-t \xi, \xi)\right)+\int_{0}^{t} \Phi^{\prime}(f) \frac{M[f]-f}{\varepsilon}(t-s, x-s \xi, \xi) d s \\
\Phi(f) \in C\left([0, T], L^{1}(\mathbb{R} \times \mathbb{R})\right),
\end{gathered}
$$


$\varphi_{\delta}(f) \rightarrow \Phi(f)$ in $C\left([0, T], L^{1}(\mathbb{R} \times \mathbb{R})\right)$, and

$$
\partial_{t} \Phi(f)+\xi \partial_{x} \Phi(f)=\Phi^{\prime}(f) \frac{M[f]-f}{\varepsilon} .
$$

For the last term $\psi\left(f_{0}\right)=f_{0}^{1+1 / \lambda} /(1+1 / \lambda)$ in (5.8), we can use the approximation

$$
\psi_{\delta}\left(f_{0}\right)=\left\{\begin{array}{l}
\psi\left(f_{0}\right) \quad \text { if } \quad 0 \leqslant f_{0} \leqslant 1 / \delta \\
\psi\left(\frac{1}{\delta}\right)+\psi^{\prime}\left(\frac{1}{\delta}\right)\left(f_{0}-\frac{1}{\delta}\right) \quad \text { if } \quad f_{0} \geqslant 1 / \delta .
\end{array}\right.
$$

Then the argument is similar as above (even simpler, $\psi^{\prime}\left(f_{0}\right) M_{0}[f] \in L^{1}$ is obtained directly since $\left.f_{0}^{1 / \lambda} \in L^{\lambda(1+1 / \lambda)}, M_{0}[f] \in L^{1+1 / \lambda}\right)$, and we do not repeat the proof.

\subsection{Proof of Theorem 1.3}

According to the previous subsections, we can apply Lemma 5.2 to each term of the decomposition (5.8). Noticing that the convention on the value of $H^{\prime}(0, \xi)$ corresponds to $\Phi^{\prime}(0)=0$, we obtain (1.25)-(1.28). Moreover, $\xi H(f(t, x, \xi), \xi) \in L^{1}(] 0, T\left[\times B_{R} \times \mathbb{R}\right)$ and

$$
\begin{gathered}
\partial_{t} \int_{\mathbb{R}} H(f, \xi) d \xi+\partial_{x} \int_{\mathbb{R}} \xi H(f, \xi) d \xi=\frac{1}{\varepsilon} \int_{\mathbb{R}} H^{\prime}(f, \xi) \cdot(M[f]-f) d \xi \\
\frac{d}{d t} \iint_{\mathbb{R} \times \mathbb{R}} H(f, \xi) d x d \xi=\frac{1}{\varepsilon} \iint_{\mathbb{R} \times \mathbb{R}} H^{\prime}(f, \xi) \cdot(M[f]-f) d x d \xi
\end{gathered}
$$

But by Proposition 2.6,

$$
P(t, x, \xi) \equiv\left(H^{\prime}(f, \xi)-\left(\frac{\gamma \kappa}{\gamma-1} \rho^{\gamma-1}-\frac{u^{2}}{2}, u\right)\right) \cdot(f-M[f]) \geqslant 0 \quad \text { a.e. },
$$

and since $\int(f-M[f]) d \xi=0$,

$$
\iiint_{] 0, T[\times \mathbb{R} \times \mathbb{R}} P(t, x, \xi) d t d x d \xi=\iiint_{] 0, T[\times \mathbb{R} \times \mathbb{R}} H^{\prime}(f, \xi) \cdot(f-M[f]) d t d x d \xi<\infty,
$$

thus $P \in L^{1}(] 0, T[\times \mathbb{R} \times \mathbb{R})$. Finally, since

$$
\int_{\mathbb{R}} P(t, x, \xi) d \xi=\int_{\mathbb{R}} H^{\prime}(f, \xi) \cdot(f-M[f]) d \xi \quad \text { a.e. } t, x,
$$

from (5.31) and (5.32) we obtain (1.29) and (1.30). 
Remark 5.1. - It is possible to prove directly that

$$
\left(\frac{\gamma \kappa}{\gamma-1} \rho^{\gamma-1}-\frac{u^{2}}{2}, u\right) \cdot(M[f]-f) \in L^{\infty}(] 0, T\left[, L^{1}(\mathbb{R} \times \mathbb{R})\right) .
$$

Indeed by (2.4) and Proposition 2.5,

$$
\frac{1}{2} \rho u^{2}+\frac{\kappa}{\gamma-1} \rho^{\gamma}=\eta(\rho, u) \leqslant \int H(f, \xi) d \xi
$$

thus

$$
\int_{\mathbb{R}}\left(\frac{1}{2} \rho u^{2}+\frac{\kappa}{\gamma-1} \rho^{\gamma}\right) d x \leqslant C_{H}^{0},
$$

and $\rho u^{2}, \rho^{\gamma} \in L^{\infty}(] 0, \infty\left[, L^{1}(\mathbb{R})\right)$. Then, the inequality

$$
\begin{aligned}
& \left(\iint\left|u(t, x) f_{1}(t, x, \xi)\right| d x d \xi\right)^{2} \\
\leqslant & \left(\iint u(t, x)^{2} f_{0}(t, x, \xi) d x d \xi\right) \times\left(\iint_{f_{0}(t, x, \xi)>0} \frac{f_{1}(t, x, \xi)^{2}}{f_{0}(t, x, \xi)} d x d \xi\right)
\end{aligned}
$$

proves that $\left\|u f_{1}\right\|_{L^{1}(\mathbb{R} \times \mathbb{R})} \leqslant 2 C_{H}^{0}$. A similar estimate holds for $u M_{1}[f]$, and the other terms in (5.36) are easy to estimate with (5.38).

\section{Bibliography}

[1] Aregba-Driollet (D.), Natalini (R.). - Discrete kinetic schemes for multidimensional systems of conservation laws, SIAM J. Numer. Anal. 37 (2000), 1973-2004.

[2] Berthelin (F.), Bouchut (F.). - Kinetic invariant domains and relaxation limit from a BGK model to isentropic gas dynamics, to appear in Asymptotic Analysis.

[3] Bouchut (F.). - Construction of BGK models with a family of kinetic entropies for a given system of conservation laws, J. Statist. Phys. 95 (1999), 113-170.

[4] BRENIER (Y.). - Averaged multivalued solutions for scalar conservation laws, SIAM J. Numer. Anal. 21 (1984), 1013-1037.

[5] Brenier (Y.), Corrias (L.). - A kinetic formulation for multi-branch entropy solutions of scalar conservation laws, Ann. Inst. H. Poincaré Anal. Non Linéaire 15 (1998), 169-190.

[6] Chen (G.Q.), Levermore (C.D.), Liu (T.-P.). - Hyperbolic conservation laws with stiff relaxation terms and entropy, Comm. Pure Appl. Math. 47 (1994), 787-830.

[7] DiPerna (R.J.). - Convergence of the viscosity method for isentropic gas dynamics, Comm. Math. Phys. 91 (1983), 1-30.

[8] Golse (F.), Lions (P.-L.), Perthame (B.), Sentis (R.). - Regularity of the moments of the solution of a transport equation, J. Funct. Anal., 76, (1988), 110-125. 
[9] Jin (S.), XIN (Z.-P.). - The relaxation schemes for systems of conservation laws in arbitrary space dimensions, Comm. Pure Appl. Math. 48 (1995), 235-276.

[10] Lions (P.-L.), Perthame (B.), Souganidis (P.E.). - Existence and stability of entropy solutions for the hyperbolic systems of isentropic gas dynamics in Eulerian and Lagrangian coordinates, Comm. Pure Appl. Math. 49 (1996), 599-638.

[11] Lions (P.-L.), Perthame (B.), Tadmor (E.). - A kinetic formulation of multidimensional scalar conservation laws and related equations, J. Amer. Math. Soc. 7 (1994), 169-191.

[12] Lions (P.-L.), Perthame (B.), Tadmor (E.). - Kinetic formulation of the isentropic gas dynamics and $p$-systems, Comm. Math. Phys. 163 (1994), 415-431.

[13] Natalini (R.). - A discrete kinetic approximation of entropy solutions to multidimensional scalar conservation laws, J. Diff. Eq. 148 (1998), 292-317.

[14] NAtAlini (R.). - Recent results on hyperbolic relaxation problems. Analysis of systems of conservation laws (Aachen, 1997), 128-198. Chapman \& Hall/CRC Monogr. Surv. Pure Appl. Math., 99, Chapman \& Hall/CRC, Boca Raton, FL, 1999.

[15] Perthame (B.). - Global existence to the BGK model of Boltzmann equation, J. Diff. Eq. 82 (1989), 191-205.

[16] Perthame (B.), Pulvirenti (M.). - Weighted $L^{\infty}$ bounds and uniqueness for the Boltzmann BGK model, Arch. Rational Mech. Anal. 125 (1993), 289-295.

[17] Perthame (B.), Tadmor (E.). - A kinetic equation with kinetic entropy functions for scalar conservation laws, Comm. Math. Phys. 136 (1991), 501-517.

[18] Serre (D.). - Relaxation semi-linéaire et cinétique des systèmes de lois de conservation, Ann. Inst. H. Poincaré Anal. non linéaire 17 (2000), 169-192. 